The Egyptian International Journal of Engineering Sciences \& Technology, Vol 8, No 1 (2004)

\title{
Performance Optimization of the High Speed Distributed Packet Switching Local Computer Network
}

Ibrahim ElEmary

\begin{abstract}
In this paper, we present and describe the topology and structure of the high speed distributed packet switching local computer network under P-persistent CSMA/CD as access protocol for the communication stations. Also, we discuss and analyze performance optimization of this network based on some analytical treatment to the applied access protocol.
\end{abstract}

